\title{
Peter Isépy
}

\section{Fragmente aus dem „Pandektes“ des Antiochos Monachos in der Palimpsesthandschrift Collegio Greco 2}

Mit Tafeln 1-3

\begin{abstract}
Codex 2 of the Greek manuscript collection of the Pontificio Collegio Greco in Rome, copied around the end of the first quarter of the 12th c., contains the commentary of Gregory of Corinth $(1070-1156)$ on the Canons of Kosmas the Hymnographer and John of Damascus on the main festivals of the Byzantine ecclesiastical year. At the end of the manuscript we find ten palimpsest leaves originally written in the 9 th/10th $c$. in a „maiuscola ogivale inclinata“. Up to now, only a general identification of the scriptio inferior with the Pandectes Scripturae Sacrae of Antiochos Monachos (7th c.) has been established. The present article aims to determine the exact extension of the „Pandectes“ transmitted in fragmentary form in the Coll. gr. 2 and offers a reconstruction of the original leaf order in the codex antiquior.
\end{abstract}

Adresse: Dr. Peter Isépy: Institut für Griechische und Lateinische Philologie, Ludwig-Maximilians-Universität München, Geschwister-Scholl-Platz 1, 80539 München, DEUTSCHLAND; peter.isepy@klassphil.uni-muenchen.de

In der 22 griechische Kodizes umfassenden Sammlung des Pontificio Collegio Greco in Rom ${ }^{1}$ nimmt die 147 Folien zählende Pergamenthandschrift Coll. gr. $2^{2}$

Der vorliegende Beitrag entstand während eines Aufenthalts im Griechischen Kolleg im Herbst 2013. Dem Rektor des Instituts, P. Archim. Manuel Nin OSB und Bibliothekar P. Lambert Vos OSB sei vielmals für den freien Zugang zum Archiv und den Kodizes des Kollegs gedankt. Mein Dank gilt zudem zwei anonymen Gutachtern für ihre äußerst wertvollen Hinweise. ACGr = Archivio del Pontificio Collegio Greco. Die Fotografien der Tafeln wurden nach freundlicher Genehmigung durch das Collegio Greco vom Verfasser erstellt.

1 Die Kodizes Nr. 1-21 der Sammlung des griechischen Kollegs wurden summarisch be-

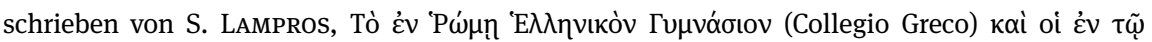

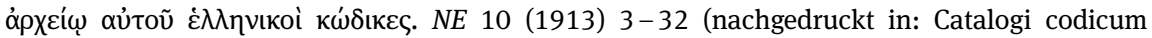
Graecorum qui in minoribus bibliothecis Italicis asservantur. In 2 vol. collati et novissimis 
eine besondere Rolle ein: Zum einen ist sie einer der ältesten Textzeugen für den Kommentar des Gregorios Pardos ( 1070 -1156), Metropolit von Korinth, zu den Kanones des Kosmas Hymnographos und Johannes von Damaskus zu den Hauptfesten des byzantinischen Kirchenjahres. ${ }^{3}$ Die Folien $1-137^{\mathrm{v}}$ des Kodex sind

additamentis aucti, accuravit C. SAMBERGER. Lipsiae 1965 - 68, II, 255 - 284). Während eine um 1950 hinzugekommene 22. Handschrift zur Kollektion gezählt wird (vgl. S. VoIcU, Un canone penitenziale dal ms. 22 del Collegio Greco di Roma, in A. Fyrigos [ed.], Il Collegio Greco di Roma, Ricerche sugli alunni, la direzione, l'attività. Analecta Collegii Graecorum, 1. Roma 1983, 351-362 und 401-402, 351), werden die nominellen Kodizes 23-36 (Aufzeichnungen von Alumnen des Collegio Greco aus dem 17.-20. Jh.s verschiedenen Inhalts, viele ohne feste Bindung) traditionell nicht als Teil des ,Hauptbestandes“ angesehen; sie wurden überblicksweise beschrieben von M. Phoskolos, Catalogo dei Manoscritti del Collegio Greco di Sant'Atanasio in Roma, Roma 1973 (unveröffentlicht, interne Signatur: ACGr 403). Zur Geschichte des 1577 von Papst Gregor XIII. gegründeten Päpstlichen Instituts vgl. u.a. R. NETZHAMmer, Das Griechische Kolleg in Rom. Salzburg 1905; P. DE MeEster, Le Collège Pontifical Grec de Rome. Rome 1910; und V. Peri, Inizi e finalità ecumeniche del Collegio Greco in Roma. Aevum 44 (1970) 1-71. Zum ehemaligen Handschriftenbestand des Kollegs (62 Kodizes, heute unter den Vat. gr. 1487-1559, in der älteren Nummerierung die Nummern 1490-1549 und 1558 - 59) und seiner Überführung in die Vaticana im Jahre 1613 vgl. P. BATIFFoL, La Vaticane depuis Paul III. Revue des Questions Historiques 45 (1889) 177 - 218 und P. CANART, Les Vaticani Graeci 1487-1962. Città del Vaticano 1979, 34f., 141-143 u. 163-190.

2 Nach einer Neubindung und Restaurierung der meisten Kodizes des ,Hauptbestandes‘ in der ersten Hälfte des letzten Jh.s sind viele der kodikologischen Angaben von Lampros, so auch im Falle des Coll. gr. 2 bezüglich der damaligen Vor- und Rücksatzblätter (heute jeweils nur eines) und der Folienzahl (heute 147), nicht mehr zutreffend. Neben der im Folgenden zitierten einschlägigen Literatur zum Coll. gr. 2 (siehe v. a. Anm. 4 und 5) wird die Handschrift am Rande auch erwähnt bei S. Venezia, Fra rinascita e declino. Dinamiche economiche e attività culturale in un monastero italo-greco siciliano dal XII al XVI secolo. BollGrott 3 (2006) 243 - 273, 250 mit Anm. 68; S. LuCÀ, Dalle collezioni manoscritte di Spagna: libri originari o provenienti dall'Italia greca medievale. RSBN 44 (2007) 39-96, 89; S. LUCÀ/S. VenezIA, Frustuli di manoscritti greci a Troina in Sicilia. Erytheia 31 (2010) 75 -132, 90 Anm. 63 sowie 115 f. Anm. 173; S. LuCÀ, Doroteo di Gaza e Niceta Stetato. A proposito del Neap. gr. 7, in R. Gentile Messina (ed.), Bisanzio e le periferie dell'impero. Atti del Convegno internazionale nell'ambito delle celebrazioni del millenario della fondazione dell'Abbazia di San Nilo a Grottaferrata (Catania, 26-28 novembre 2007). Acireale 2011, 145 180, 169, Anm. 93 und S. LuCÀ, Una donazione al monastero dei SS. Pietro e Paolo di Arena, in Calabria (1184-1185), in P. Cherubini/G. Nicolaj (ed.), Sit liber gratus quem est operatus. Studi in onore di Alessandro Pratesi per il suo 90 compleanno. Littera antiqua, 19. Tomo I. Città del Vaticano 2012, 317 -336, 324, Anm. 43.

3 Zur Person des Gregorios vgl. A. Kazhdan, s.v. Pardos, Gregory. ODB 1587. Der Kommentar des Gregorios wurde teilweise herausgegeben von F. MontanA, Gregorio di Corinto, Esegesi al canone giambico per la Pentecoste attribuito a Giovanni Damasceno, Introduzione, edizione critica, traduzione. Pisa 1995, bes. XIVf. Der Kodex Coll. gr. 2, mit der Sigle C bezeichnet, stammt zusammen mit den Handschriften Vat. gr. 2078 (Va) und Vind. Theol. gr. 121 (Wa) von der verlorenen Stufe $\alpha^{1}$ ab (vgl. MonTAna XXXII ff.). 
gegen Ende des ersten Viertels des 12. Jh.s im sog. „Reggio-Stil“ von dem $\kappa \alpha \lambda \lambda_{\mathrm{t}}$ yрá $\varphi$ о Leon von Reggio, tätig in Troina (Sizilien), geschrieben worden. Dies zeigt der Vergleich mit dem vom selbigen Schreiber 1124/25 vollendeten Vat. gr. 1926, der lückenhaft ebenfalls die Auslegungen Gregors überliefert. ${ }^{4}$ Ein weiterer Grund für die Bedeutung des Kodex beruht auf der letzten Lage der Handschrift, einem palimpsestierten Quinio, der die fol. $138^{\mathrm{r}}$ bis $147^{\mathrm{v}}$ umfasst: Während die scriptio superior, nicht mehr von der Hand Leons, ${ }^{5}$ den Kommentar des Gregorios Pardos fortführt, ${ }^{6}$ sieht man sich im Falle der scriptio inferior einer „maiuscola ogivale inclinata“ des 9./10. Jh.s gegenüber, die Teile des sog. „Pandektes“ des Antiochos Monachos überliefert.

Unabhängig von einer möglicherweise späteren Ausführung der scriptio superior der letzten Lage (vgl. oben Fußnote 5) bedient sich ihr Schreiber jedenfalls einer Minuskel, die zwar grundsätzlich versucht, die Charakteristika des „Reggio-Stiles“ zu wahren, dabei aber von Anfang an das hohe kalligraphische Stilisierungsniveau Leons verlässt und unordentlicher und roher erscheint. So findet man zwar die typische Gegenüberstellung der breiten und schmalen Buchstaben sowie die charakteristischen Formen einzelner Buchstaben und Ligaturen, ${ }^{7}$ wie beispielsweise das Minuskel-Gamma mit erster, vertikaler Haste (fol. $138^{\mathrm{r}}$, 8. Z.), Zeta in 3-Form (fol. $143^{\mathrm{r}}$, z. B. 1. u. 5. Z.),

4 Vgl. S. LuCÀ, Teodoro Sacerdote, copista del Reg. gr. Pii II 35. Appunti su scribi e committenti di manoscritti greci. BollGrott 55 (2001) 127 - 163, 141; P. CANART, Codices Vaticani Graeci 1745 1962, I. Città del Vaticano 1970, 689 ff.; S. LuCÀ, Il Vaticano greco 1926 e altri codici della biblioteca dell'Archimandritato di Messina. Schede Medievali 8 (1985) 51-79, 52 ff.; und S. LucÀ, I Normanni e la ,rinascita' del sec. XII. Archivio storico per la Calabria e la Lucania 60 (1993) 47, Anm. 183. Eine um vieles frühere Entstehung des Coll. gr. 2 macht der terminus post quem für die Entstehung des Kommentars nach 1110 (vgl. MontanA, wie oben Fußnote 3, XLIX) und das erste (datierte) Auftreten des „stile di Reggio“ 1118 (vgl. P. CANART / J. LEROY, Les manuscrits en style de Reggio. Étude paléographique et codicologique, in: La paléographie grecque et byzantine. Colloques internationaux du CNRS, 559. Paris 1977, 241 - 261, 243 und 256) unwahrscheinlich. Eine spätere Entstehung ist grundsätzlich nicht auszuschließen. Zum „Reggio-Stil“, der seine Blütezeit im 12. Jh. erlebt, doch bis zum Beginn des 14. Jh.s überlebt, vgl. CANART/LERoY ebd. 241-261 und E. CRISCI / P. DEGNI, La scrittura greca dall'antichità all'epoca della stampa. Una introduzione. Roma 2011, 172-174. Zur Person von Leon, den Charakteristika seiner Hand und für eine Abbildung aus dem Vat. gr. 1926 vgl. E. GAMILLSCHEG / D. HARLFINGER/H. HUNGER, Repertorium der griechischen Kopisten 800 - 1600, 3. Teil: Handschriften aus Bibliotheken Roms mit dem Vatikan, A/B/C. Wien 1997, Nr. 390 bzw. Taf. 216. Da der Vaticanus die Schrift Gregors heute nur noch bruchstückhaft wiedergibt, wurde er von Montana nicht zur Edition hinzugezogen (vgl. MoNTANA, wie oben Fußnote 3, XIIf.).

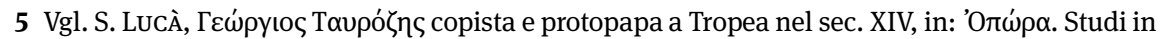
onore di Mgr Paul Canart per il LXX compleanno, III = BollGrott 53 (1999) 285-347, 315 f. Anm. 118, der davon ausgeht, dass der mutilierte Kodex erst im Verlauf des 13. Jh.s um die fol. $138^{\mathrm{r}}-147^{\mathrm{v}}$ erweitert wurde.

6 Er endet auf fol. $147^{\mathrm{r}}$ mit dem Kanon des Kosmas zur Entschlafung bzw. dessen Auslegung. Die zweite Hälfte des fol. $147^{\mathrm{r}}$ und das ganze Verso sind von der scriptio superior frei.

7 Vgl. CANART/LERoY, wie oben Fußnote 4, $244 \mathrm{ff}$. 
die Ligatur $\alpha-\varphi$ (fol. 138 ${ }^{\mathrm{r}}$, 13.-15. Z.) oder auch das Kürzel für kaí (fol. 138 r , 6. u. 10. Z. v.u.). Doch weicht nicht nur der luftige Zeilenabstand der fol.1-137 (Schriftspiegel: ca. $200 \times 130 \mathrm{~mm}$, 26 Z. pro Seite) einem gedrängteren Schriftbild (Schriftspiegel: ca. $190 \times 145 \mathrm{~mm}$, ca. 24-28 Z. pro Seite); auch gibt es mehrere Zeichen für das ,Ausbrechen' des Schreibers aus festen Strukturen: Dazu zählt v.a. die Vergrößerung einzelner Buchstaben (v.a. Gamma, Tau, Lambda und Zeta), die Verwendung von herausstechenden Buchstabenformen (vgl. v.a. das nach links gekippte Majuskel- $\alpha$ in Form einer Lanzenspitze, z. B. fol. 143 ${ }^{\text {r }}$, 6. Z. v.u.) und die Tatsache, dass der Kopist am Ende der Seiten, v. a. auf fol. $140^{v}$ f., die Unterlängen der Buchstaben teilweise weit in den Freirand hineinzieht. Wenn auch einige Initialen durch dickere Strichführung, Vergrößerung und Ekthesis hervorgehoben werden, verzichtet der Kopist völlig auf Verzierungen und Rubrizierungen, die maßgeblich zum eleganten Ausdruck der durch Leon ausgeführten Seiten beitragen. ${ }^{8}$ Der Schreiber der fol. $138 \mathrm{ff}$. verwendete außerdem eine dunklere Tinte als Leon, fast schwarz, offenbar, damit die scriptio superior auf dem durch mehrfache Bearbeitung verdunkelten Pergament noch gelesen werden konnte.

Es ist bekannt, dass schon Angelo Mai (1782-1854) die Handschriften des Griechischen Kollegs untersucht und dabei die folia rescripta des Coll. gr. 2 mit Chemikalien behandelt hat. ${ }^{9}$ Inwieweit Mai jedoch zu einer Identifizierung des Inhalts gelangte, ist unklar. ${ }^{10}$ Später, im Jahre 1913, spricht jedenfalls Spyridon Lampros in seiner wenige Zeilen umfassenden Beschreibung der Handschrift von der scriptio

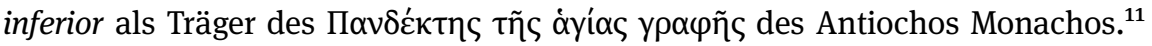
Antiochos, mit dem Epitheton „Pandektes““, ${ }^{12}$ war Mönch des Mar Saba-Klosters

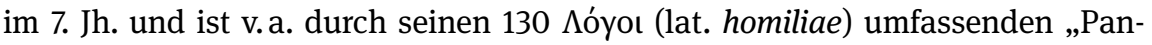

8 Die Überschriften der Kanones bzw. Auslegungen zu den Festen wurden von Leon i.d.R. von einer sehr einfachen, geschlängelten Zierlinie überdacht (z. B. fol. $55^{\mathrm{r}}$, Kanon des Kosmas zum Karfreitag; vgl. CANART/Leroy, wie oben Fußnote 4, 261, Fig. 7). Eine Ausnahme macht der Kanon des Kosmas zum Palmsonntag, dem auf fol. 20v eine breite Zierleiste aus dem Bereich der „Rankenornamentik“ (rinceau) vorangeht, die dem Typ ,a viticcio“ zugeordnet werden kann (vgl. M.L. AGati, Il libro manoscritto da oriente a occidente, Per una codicologia comparata. Roma 2009, 320 f.). Sie ähnelt dem bei Agati in Figur 88, XIII a angeführten Beispiel (vgl. Taf. 1). Neben

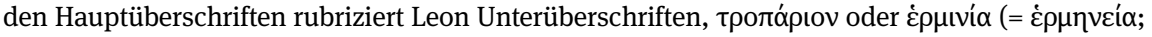
z. B. fol. $45^{\mathrm{v}} \mathrm{f}$.), und die Initialen jedes Absatzes sind i.d. R. durch Ekthesis hervorgehoben.

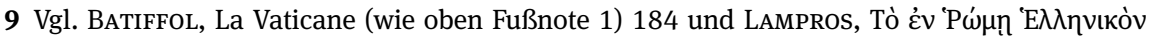
Гuнváoı

10 Über die Ergebnisse Mais bzw. den Verbleib seiner von Lampros ohne nähere Angabe er-

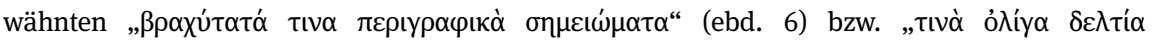

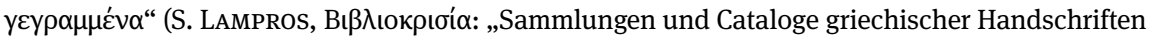

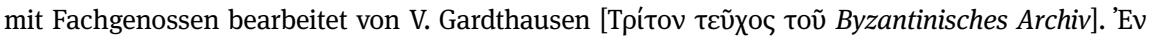

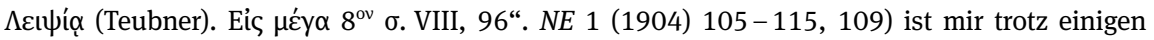
Suchens nichts bekannt.

11 Lampros (wie oben Fußnote 1) 8.

12 Die Abgrenzung von bzw. Gleichsetzung mit Antiochos Strategos ist umstritten; vgl. A. KazHDAN, s.v. Antiochos Strategos. ODB 119 f. und H.-G. BECK, Kirche und theologische Literatur im byzantinischen Reich. München 1959, 449. 
dektes" bekannt, ein auf der Hl. Schrift und den Vätern basierendes Handbuch des geistlichen Lebens. ${ }^{13}$ Die Schrift wurde gewisse Zeit nach 622 auf Bitte von Eustathios, Abt des Attaline-Klosters von Ankara, abgefasst. Wie aus dem Antwortschreiben des Antiochos an Eustathios hervorgeht, sollte der „Pandektes“ als ,allumfassendes‘ Kompendium der Väter und der Hl. Schrift gleichsam als Ersatz einer Klosterbibliothek für Eustathios’ Mönche dienen, die sich nach der Verwüstung Ankaras auf der Flucht befanden. Neben dieser Epistula ad Eustathium und dem daran anschließenden „Pandektes“ ist die Exomologesis, eine Klage über die Einnahme des Heiligen Landes durch die Perser, als Werk des Antiochos überliefert, das im 89. Band der Patrologia Graeca zu finden ist. ${ }^{14}$

Bei dem von Lampros identifizierten Passus der scriptio inferior im Coll. gr. 2 auf fol. $142^{\mathrm{v}}$ handelt es sich um den Anfang des 114. Logos aus dem „Pandektes“,

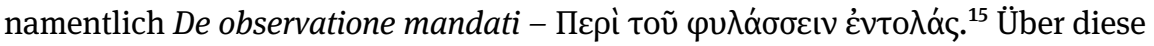
punktuelle Identifizierung hinaus hat Sever Voicu in einer unveröffentlichten, daktylographischen Beschreibung des Kodex notiert: ${ }^{16}$ „I ff. 138-147 sono palinsesti. La scrittura inferiore contiene le omelie di Antioco Monaco (M. G. 89). La parte finora identificata presenta frammenti delle omelie 107-116.“

Die bisher also nur punktuell oder ungenau erfolgte Identifizierung gibt weder Auskunft über den exakten Umfang des „Pandektes“-Fragmentes, noch ist die Möglichkeit ausgeschlossen, dass auf den anderen palimpsestierten Folien Reste eines anderen Textes verborgen geblieben sind.

Die im Rahmen der Neukatalogisierung der Handschriften des Griechischen Kollegs durch den Verfasser aufgenommenen Arbeiten am Coll. gr. 2 haben nun kürzlich zur genauen Identifizierung der durch die scriptio inferior auf den fol. $138^{\mathrm{r}}-147^{\mathrm{v}}$ überlieferten Textpartien geführt: Es handelt sich - ganz oder in Teilen um die $\Lambda$ óyo 75 bis 78 sowie 106 bis 116 des „Pandektes“ des Antiochos Monachos. Zwar lässt sich die Schrift nur auf einigen Seiten mit bloßem Auge entziffern, doch ermöglichte die Kombination des Textes der lesbaren Passagen mit der materiellen Zusammengehörigkeit der Seiten sowohl die wortgenaue Bestimmung des Umfangs des überlieferten Textes als auch die Rekonstruktion der ursprünglichen Abfolge der Blätter im einstigen codex antiquior.

13 Vgl. Beck, Kirche (wie oben Fußnote 12) 449 und P. HatliE, A rough-guide to Byzantine monasticism in the early seventh century, in G.J. Reinink/B.H. Stolte (Hrsg.), The reign of Heraclius (610-641). Leuven 2002, 205-226, $205 \mathrm{ff}$.

14 PG 89, Epistula 1421-1428, „Pandektes“ 1427-1850, Exomologesis 1849-1856.

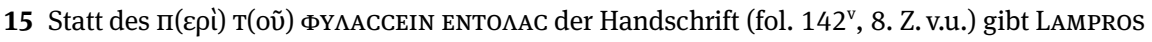

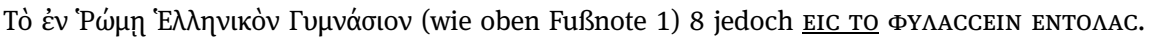
16 Sever Voicu sei vielmals für die freundliche Genehmigung gedankt, seine im Collegio Greco aufbewahrten Aufzeichnungen in neue Arbeiten zu den Kodizes miteinbeziehen zu dürfen. 
Zunächst sei auf einige paläographische Charakteristika der scriptio inferior hingewiesen. Wir sehen uns einer „maiuscola ogivale inclinata“ in zweispaltiger Ausführung mit durchschnittlich je 49 Zeilen gegenüber. Schon die sichere Herkunft Leons - und wohl auch des Kopisten der scriptio superior der letzten Lage aus Süditalien macht es sehr plausibel, dass auch die Entstehung der Majuskelschrift ebendort zu verorten ist. Dafür spricht nun aber auch, dass die hier vorliegende nach rechts geneigte „Spitzbogenmajuskel“ alle Eigenheiten aufweist, die sonst für den süditalienischen Typ dieser Schrift angeführt wurden. ${ }^{17}$ Insbesondere zeigt die Schrift eine deutliche Ähnlichkeit mit einer Gruppe von Handschriften, die Edoardo Crisci im Rahmen seiner Arbeit an den Palimpsesthandschriften von Grottaferrata benannt hat. ${ }^{18}$ Dazu gehören die Zeugnisse aus dem 9.

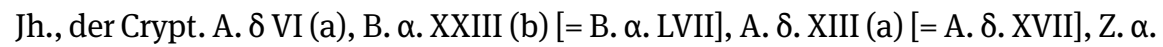
XXIV (a) und der Vat. gr. 2066 + Washington, Library of Congress $60^{19}$ wie auch der Vat. gr. 1589 aus dem 10. Jh., die sich allesamt durch kleine Buchstabenkörper, einen unregelmäßigen Neigungswinkel, inkonsequente Dicke der Haar- und Schattenstriche und eine gewisse Einfachheit bis Grobheit im Gesamteindruck, unterstrichen durch die wenig akkuraten Ornamentierungen, auszeichnen. ${ }^{20}$ Wenn der Coll. gr. 2 auch weniger roh erscheint und nicht zuletzt durch rubrizierte Überschriften und leicht verzierte Initialen (vgl. z. B. das H auf fol. 143') ein gewisses repräsentatives Element bezeugt, ist in den anderen Punkten die Ähnlichkeit nicht zu übersehen. Eine Entstehung im 8. Jh., wie von Lampros vorgeschlagen, ist nicht nur deshalb sicher zu früh angesetzt. Auch aufgrund der -

17 Vgl. z. B. G. CAVALlo, Funzione e strutture della maiuscola greca tra i secoli VIII-XI, in: La paléographie grecque et byzantine (wie oben Fußnote 4), 95-137, 100 ff.; DERS., Le tipologie della cultura nel riflesso delle testimonianze scritte, in: Bisanzio, Roma e l'Italia nell'alto medioevo (Spoleto, 3 -9 aprile 1986). Settimane di studio del Centro italiano di studi sull'alto medioevo, 34. Spoleto, Centro italiano di studi sull'alto medioevo 1988, 467-516, tavv. I-LVI, 503 ff.; und E. CRISCI, I palinsesti di Grottaferrata, Studio codicologico e paleografico. Napoli $1990,281 \mathrm{ff}$.

18 Vgl. ebd. $281 \mathrm{f}$.

19 Zwei Folien der Handschrift befinden sich in Washington, vgl. dazu W. JAEGER, Greek uncial fragments in the Library of Congress in Washington (Ms 60). Traditio 5 (1947) 79-102. Zur Handschriftenbeschreibung vgl. auch E. FolLIERI, Codices graeci Bibliothecae Vaticanae selecti. Città del Vaticano 1969, 6f. mit Tab. 6. Während JAEGER $95 \mathrm{f}$. eine konstantinopolitanische Herkunft vermutet, vertreten P. BATIFFoL, L'abbaye de Rossano, contribution à l'histoire de la Vaticane. Paris 1891, 104; CaVAlLO, Funzione (wie oben Fußnote 17) 101; und CRISCI, Palinsesti (wie oben Fußnote 17) 281f. die Meinung, die Handschrift sei im Westen (Kalabrien oder gar Rom?) entstanden.

20 Vgl. CRISCI, Palinsesti (wie oben Fußnote 17) 281. 
soweit man sehen kann - konsequent gesetzten Akzente und Spiritus von erster Hand erscheint vielmehr eine Datierung ins 9./10. Jh. angemessen. ${ }^{21}$

Auf den fol. $138^{\mathrm{r}}-147^{\mathrm{v}}$ des Coll. gr. 2 umfasst die scriptio inferior folgende Textpartien: ${ }^{22}$

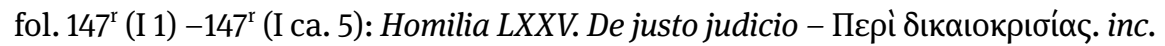

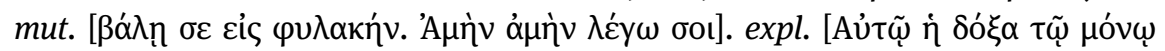

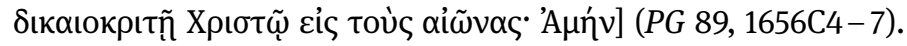

fol. $147^{\mathrm{r}}$ (I ca. 6) $-147^{\mathrm{r}}$ (II 27. Z. v.u.): Homilia LXXVI. De justa mensura - Пعрі

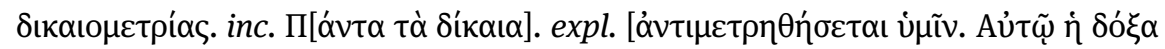

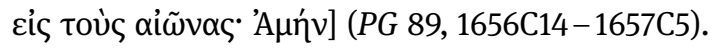

fol. $147^{\mathrm{r}}$ (II 26. Z. v.u.) $-147^{\mathrm{v}}$ und $138^{\mathrm{r}}-138^{\mathrm{v}}$ (I ca. 22. Z. v.u.): Homilia LXXVII. De

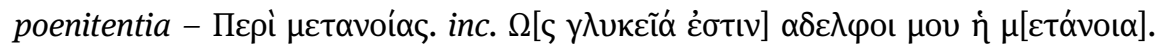

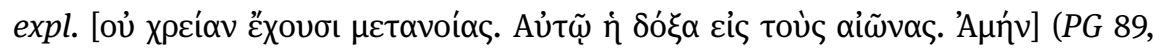
$1657 \mathrm{C} 8-1665 \mathrm{~A} 3)$

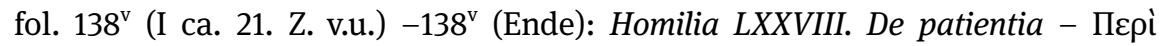

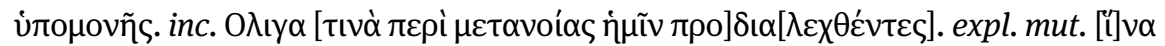
бо[ı $\beta]$ ] $[\theta] \varepsilon ı \sigma \eta^{23 .}$ (PG 89, 1665A9-1668A1).

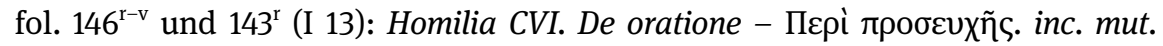

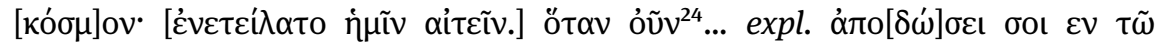

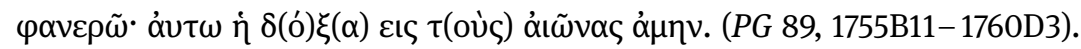

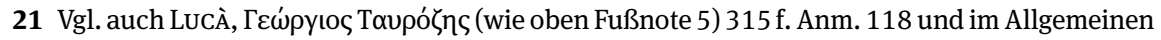
zur Akzentsetzung in Majuskelhandschriften M. REIL, Zur Akzentuation griechischer Handschriften. BZ 19 (1910) 476-529, hier 480.

22 Die Reihenfolge der Angaben ist von den Logoi des „Pandektes“, nicht der heutigen Foliierung bestimmt. Die Handschrift weicht offenbar von der Nummerierung in der $P G$ ab (sie vermerkt z. B. für die Homilie 115 auf fol. $143^{r}$ die Nummer PZ). Gemäß FolLIERI, Codices (wie oben Fußnote 19) 7 sind mit eckigen Klammern [...] diejenigen Buchstaben, Wörter oder Passagen bezeichnet, die aufgrund mechanischer Beschädigung (hier i.d.R. der Palimpestierung) fehlen bzw. mit bloßem Auge nicht entziffert oder wenigstens nachvollzogen werden konnten; sie wurden nach dem Text in $P G$ 89, 1421 ff. eingefügt. Fußnoten verweisen auf Abweichungen im Migne-Text. Mit runden Klammern (...) werden durch Kontraktion oder Suspension ausgesparte Buchstaben bezeichnet. Die Transkription bewahrt die Schreibung der Wörter in der Handschrift und gibt Akzente und diakritische Zeichen insoweit wieder, als diese mit bloßem Auge erkennbar sind.

23 ßoпөńon $P G$.

24 "Oนع oũv $P G$. 


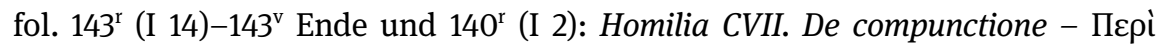

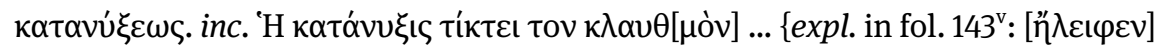

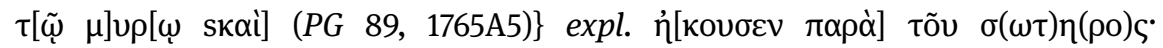

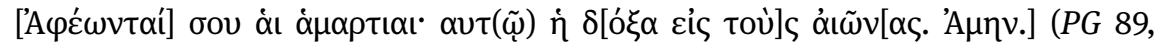
1761A1-1765A7).

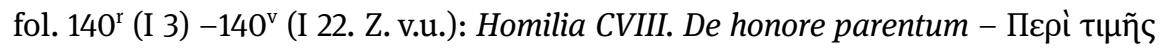

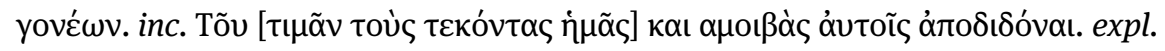

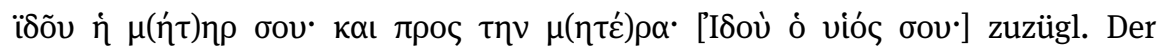

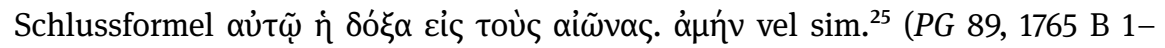
1768C9).

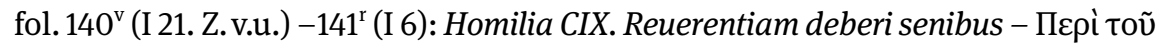

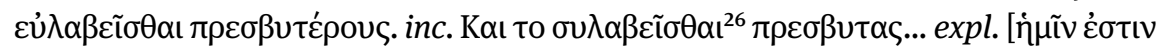

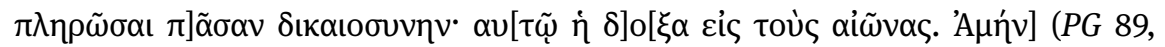
1767C14-1769C7).

fol. $141^{\mathrm{r}}$ (I 7) $-141^{\mathrm{v}}$ (II 26): Homilia CX. De longanimitate siue lenitate animi, et

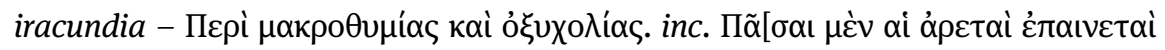

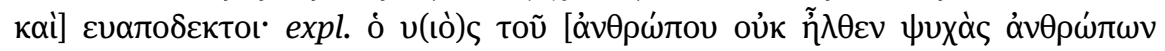

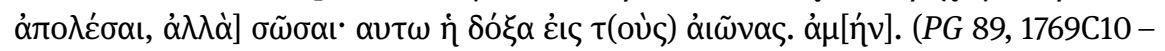
1773B12).

fol. $141^{\mathrm{v}}$ (II 27) $-141^{\mathrm{v}}$ Ende und $144^{\mathrm{r}}-145^{\mathrm{r}}$ (I Ende): Homilia CXI. De his qui praesunt -

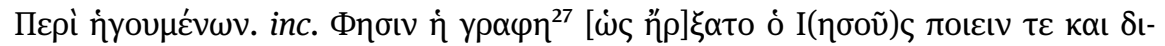

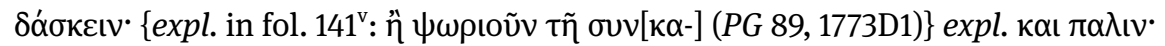
[Y

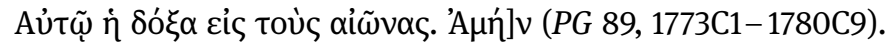

fol. $145^{\mathrm{r}}$ (II 1) $-145^{\mathrm{v}}$ Ende und $142^{\mathrm{r}}$ (I 5. Z. v.u.): Homilia CXII. De renuntiatione - Пعрі

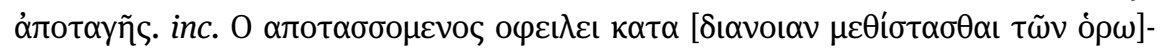

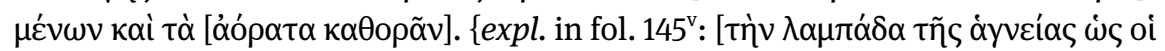

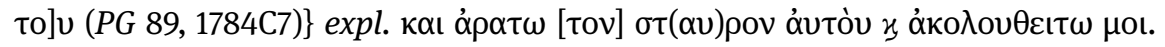

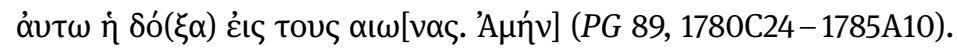

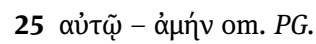

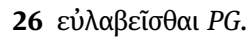

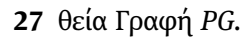


fol. $142^{\mathrm{r}}$ (I 4. Z. v.u.) -142v (I 9. Z. v.u.): Homilia CXIII. De subjectione - Пعрї ن்

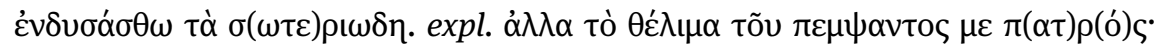

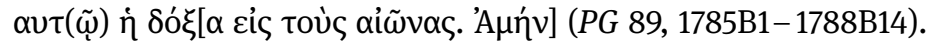

fol. $142^{\mathrm{v}}$ (I 8. Z. v.u.) $-142^{\mathrm{v}}$ Ende und 139 (II 15): Homilia CXIV. De observatione

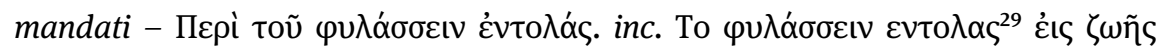

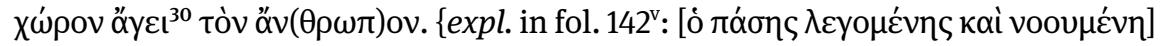

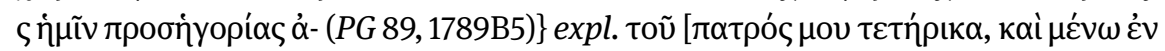

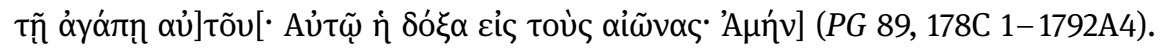

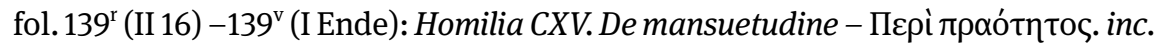

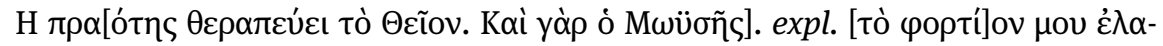

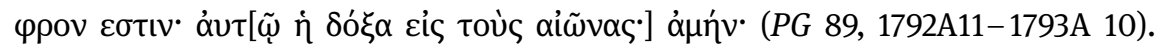

fol. $139^{v}$ (II 1) -139v (Ende): Homilia CXVI. Injuriam ferto patienter, ac nemini interim

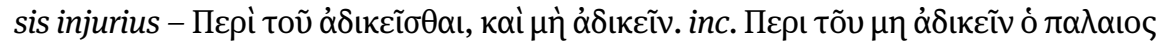

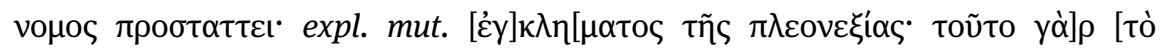
$\theta \dot{\varepsilon} \lambda \eta] \mu[\alpha](P G$ 89, 1793B1-D1).

Bei dem von der letzten Lage des Coll. gr. 2 umfassten Text des „Pandektes“ handelt es sich also um zwei zusammenhängende Textpassagen: a) auf den fol. 138+147 stehen die Homilien 75 (Ende) bis 78 (Anfang); b) auf den fol. 139-146 finden sich die Homilien 106 (Ende) bis 116 (Anfang). Wie die gerade in ihrer korrekten Abfolge aufgeführten Textausschnitte zeigen, verläuft die scriptio inferior inhaltlich jedoch keineswegs parallel zur scriptio superior bzw. gemäß der heutigen Foliierung. Das folgende Schema zeigt den Verlauf der beiden Texte zusammen, wobei die Foliierung 138-147 naturgemäß für die scriptio superior, die für diesen Zweck eingeführten Positions-Nummern 1a-10b jedoch für die scriptio inferior, d.h. den „Pandektes“, stehen:

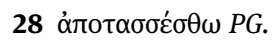

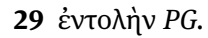

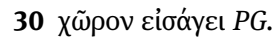




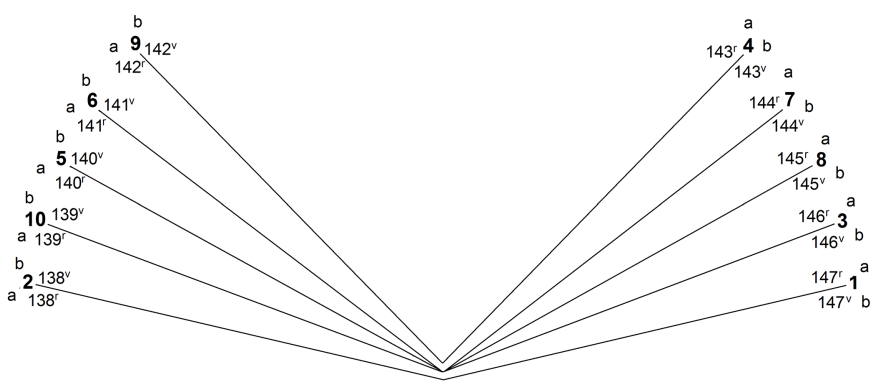

Als Ausgangspunkte für eine Rekonstruktion der kodikologischen Verhältnisse im codex antiquior können zwei - in der ersten Grafik angedeutete - Beobachtungen dienen: Zum einen ist ersichtlich, dass die innersten Folien des heutigen Quinio, die fol. 142+143, ein zusammenhängendes bifolium bilden (vgl. auch Taf. 3). Zum anderen liegt es aufgrund des Inhalts der „Textpassage a)“ (Logoi 75-78) und ihres kontinuierlichen Verlaufs auf den fol. 138+147 (in der Reihenfolge $147^{\mathrm{r}} \rightarrow$ $147^{\mathrm{v}} \rightarrow 138^{\mathrm{r}} \rightarrow 138^{\mathrm{v}}$ ) auf der Hand, dass es sich auch bei diesem ,Umschlag‘ für die anderen Blätter des aktuellen Quinio um ein bifolium handelt und es einst - als innerstes Doppelblatt - vermutlich Teil einer früheren Lage im codex antiquior war als die fol.139-146 (Logoi 106 bis 116). Wurden nun weiter bei der Konstitution des heutigen Quinio, wie zu erwarten, Doppelblätter ${ }^{31}$ ineinander geschachtelt - dies ist ferner auch dadurch gesichert, dass weder zwischen fol. 139+140 noch 140+141 (bzw. 144+145 und 145+146) eine materielle Einheit festgestellt werden kann -, so müssen auch im codex antiquior dieselben Blatt-Kombinationen vorgelegen und also die sich aktuell gegenüberliegenden Positionsnummern bifolia gebildet haben: $10-3,5-8$ und $6-7$.

Was nun aber die richtige Anordnung der bifolia in dieser Lage der ehemaligen „Pandektes“-Handschrift betrifft, so müssen schlicht die Positionsnummern 3-10 in die korrekte Reihenfolge gebracht werden. Beispielsweise mag also das bifolium fol. 142+143 zwar in seiner aktuellen Lage das innerste Doppelblatt des Quinio sein, doch hatte es im codex antiquior weder diese Innenposition noch diese Lage: Wie die Ziffern der Textabfolge, 9 und 4, zeigen, war es so gelegt bzw. derart in der Lage

31 Es ist gänzlich unwahrscheinlich und wäre ungewöhnlich, wenn bei der Konzeption des Quinio nicht nur bifolia verwendet, sondern auch Einzelblätter in den ,Umschlag‘ fol. $147+138$, Pos. 1+2, eingebunden worden wären. 
positioniert, dass der Text von fol. 142 ${ }^{\mathrm{v}}$ (Pos. 9) auf fol. 139 ${ }^{\mathrm{r}}$ (Pos. 10) weiterlaufen konnte usw. Die kodikologische Struktur der Doppelblätter im codex antiquior, auf denen die Text-Abschnitte 3-10 überliefert werden, kann also wie folgt dargestellt werden. ${ }^{32}$

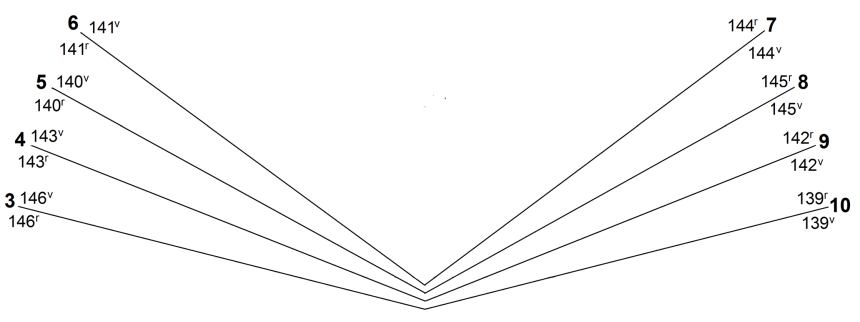

Überblickt man nun grob, wie viele griechische $P G$-Seiten bzw. -Spalten der ,Quaternio، fol. 146-139 ausmacht, so kommt man auf ca.19,4 Textspalten (PG 89, 1755B11-1793D1), was bedeutet, dass eine Seite der Handschrift den Text von durchschnittlich 1, 21 PG-Spalten umfasst (bzw. 1 PG-Spalte 0, 82 Seiten im Kodex entspricht). Da zwischen Homelie 78 (Ende; fol. 138v) und 108 (Anfang; fol. 146 ${ }^{\mathrm{r}}$ ) jedoch ca. 44,45 PG-Spalten (= ca. 36,45 Kodex-Seiten) fehlen, steht fest, dass fol. 147+138 einerseits und die übrigen Folien andererseits nicht aus benachbarten Lagen stammen können. Es lässt sich weiter vermuten, dass es sich im Falle des codex antiquior um eine Quaternionen-Handschrift gehandelt hat: Eine Kalkulation mit Quaternionen ergibt nämlich, dass sich die im Kodex zwischen den beiden angenommenen Quaternionen veranschlagte Seitenanzahl auf 1,90 Lagen verteilt, wonach also knapp zwei Lagen fehlen würden. ${ }^{33}$ Demgegenüber geht eine Rechnung mit Quinionen - nur eine Lage würde fehlen - bei weitem nicht so gut auf. $^{34}$

32 Die „Lex Gregory“ ist im Übrigen sowohl in der aktuellen Folien-Abfolge (139 ${ }^{\mathrm{r}}-146^{\mathrm{v}}$; FHHF) als auch in jener des codex antiquior (146-143-140-141-144-145-142-139; FHHF) gewahrt.

33 Von den 36, 45 Kodex-Seiten werden die 3 Folien bzw. 6 Seiten subtrahiert, die auf fol. 138 in einem angenommenen Quaternio noch folgen würden. Die 30, 45 Kodex-Seiten, die also zwischen den beiden angenommenen Quaternionen fehlen würden, lassen sich auf 1,90 Lagen aufteilen. 34 Von den 36, 45 Kodex-Seiten werden 4 Folien bzw. 8 Seiten subtrahiert, die auf fol. 138 in einem angenommenen Quinio noch folgen würden und zudem das eine Folio bzw. die 2 Seiten 
Wenn man diese Vermutungen allein auf den „Pandektes“ des Antiochos bezieht, nicht auf eventuelle weitere Werke im codex antiquior, lässt sich die ursprüngliche Situation der heute im Coll. gr. 2 befindlichen Fragmente schematisch dergestalt zusammenfassen:
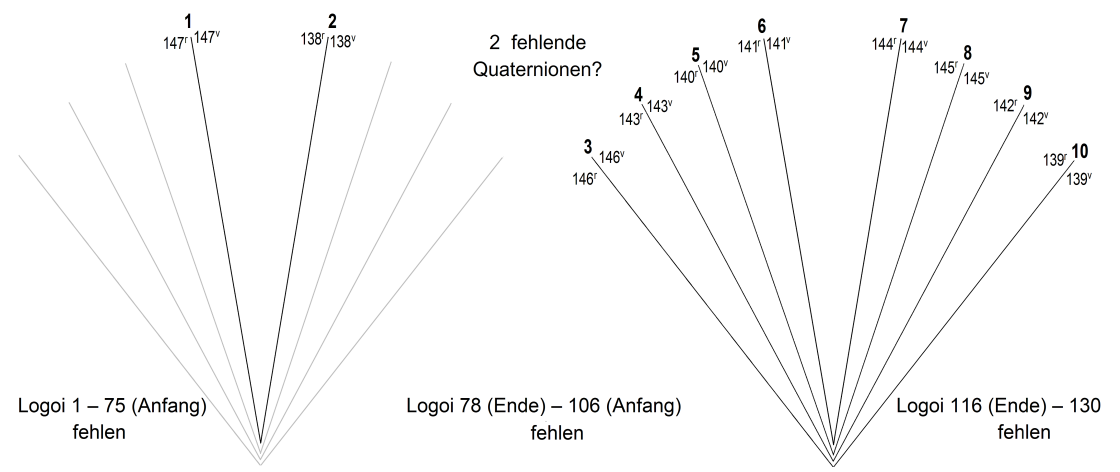

Angesichts der Tatsache, dass der Coll. gr. 2 einer der ältesten Textzeugen der „Pandektes“-Überlieferung ist, ${ }^{35}$ sollte ein zukünftiger Editor der Schrift auch den

abgezogen, die fol. 146 in einem angenommenen Quinio vorausgehen würden. Die 26, 45 KodexBlätter, die also zwischen den beiden angenommenen Quinionen fehlen würden, gehen lediglich in 1,32 Quinionen auf.

35 Vgl. http://pinakes.irht.cnrs.fr, s.v. Antiochus Monachus, Pandectes Scripturae Sacrae, wo der Coll. gr. 2 jedoch auch was den „Pandektes“ betrifft als im 12. Jh. entstanden genannt wird. Gemäß den Angaben der Datenbank stammen die Kodizes Rom, Bibl. Angelica gr. 79, Amorgos, Movì

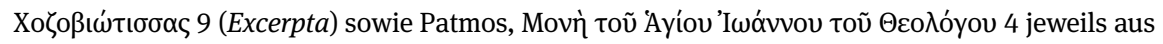
dem 9. Jh. Die Handschrift Bibl. Angelica gr. 79 ist laut der Beschreibung von E. ScIARRA in „Manus Online“ (http://manus.iccu.sbn.it//opac_SchedaScheda.php?ID= 117050; 02.10.2013) in der Tat im 9./10. Jh. entstanden und wurde ebenfalls in einer zweispaltigen, jedoch in ihrer Ausführung verschiedenen „maiuscola ogivale inclinata“ geschrieben. Die vermeintlich im 9. Jh. entstandenen Kodizes von Amorgos und Patmos sind offenbar aus späterer Zeit: Den Ersteren

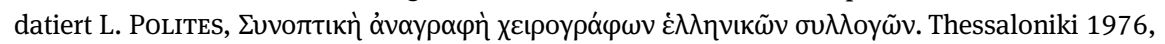

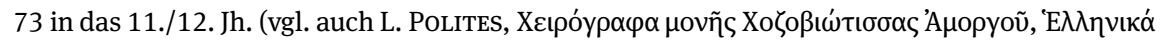
20, 1967, 486, der den Kodex Nr. 4 des Chozobiotissa-Klosters aus der 1. H. d. 10. Jh.s als den ältesten der Sammlung angibt; vgl. zu den neuen Signaturen J.-M. OLIVIER, Répertoire des bibliothèques et des catalogues de manuscrits grecs de Marcel Richard. Turnhout 1995, 77), den

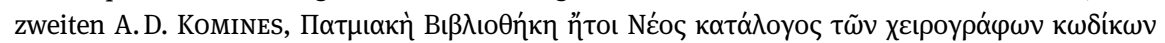

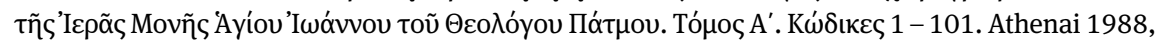
5 in das 11. Jh. Als im 10. Jh. entstanden werden genannt die Kodizes Athen, Nationalbibliothek

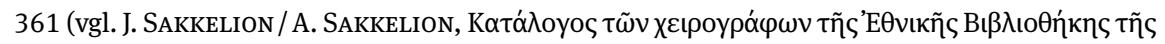

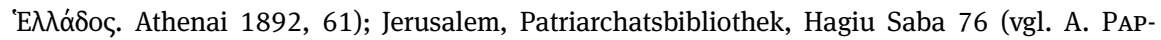


Palimpsest des Griechischen Kollegs bezüglich seiner Tauglichkeit für die Textkonstitution untersuchen. Dazu ermutigt nicht nur das Alter des Kodex, sondern auch die Passagen, die mit bloßem Auge gelesen werden können: Immerhin lassen sich dabei einige Abweichungen zum Text von Migne feststellen, ${ }^{36}$ deren Beschaffenheit vor dem Hintergrund der Lesarten der restlichen Überlieferung überprüft werden sollte. Auch die übrigen, stark verblichenen Stellen der scriptio inferior dürften unter Verwendung von UV-Licht zu entziffern sein.

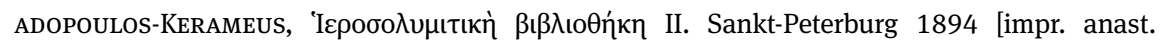
Bruxelles 1963], 136); Oxford, Bodleian Library, Auct. D.4.1 (Misc. 5) (von der Hand des Anthimos um 950, vgl. N.Wilson, Mediaeval Greek bookhands. Examples selected from Greek manuscripts in Oxford libraries. Mediaeval Academy of America, 81. 2 vols. Cambridge (Mass.) 1972 - 1973, Nr. 24 und Gamillscheg/Harlfinger/Hunger, Repertorium, wie oben Fußnote 4, 1. Teil: Handschriften aus Bibliotheken Großbritanniens, A/B/C Wien 1981, Nr. 21); Paris, Nationalbibliothek, gr. 881 (vgl. H. OMONT, Inventaire sommaire des manuscrits grecs de la Bibliothèque Nationale, Première partie, Ancien fonds grec, Théologie. Paris 1886, 165); Sankt Petersburg, Russische Nationalbibliothek, Ф. No. 906, gr. 342 (Granstrem 125) (vgl. E. GRANSTREM, Katalog grečeskich rukopisej leningradskich chranilišc. 2. VV 18 (1961) 254-274, 260); und Vaticano, Biblioteca Apostolica Vaticana, gr. 2027 (geschrieben von Theognostos im Jahre 959, vgl. K. LAKE / S. LAKE, Dated Greek minuscule manuscripts to the year 1200, VII: Manuscripts in Rome, Part I. Boston 1937, 262 bzw. Pl. 465 und GAmillscheg/Harlfinger/Hunger, Repertorium, wie oben Fußnote 4, Nr. 201 bzw. Taf. 107).

36 Vgl. oben die Fußnote 23 bis 30. 
Bereitgestellt von | Ludwig-Maximilians-Universität München Universitätsbibliothek (LMU) 